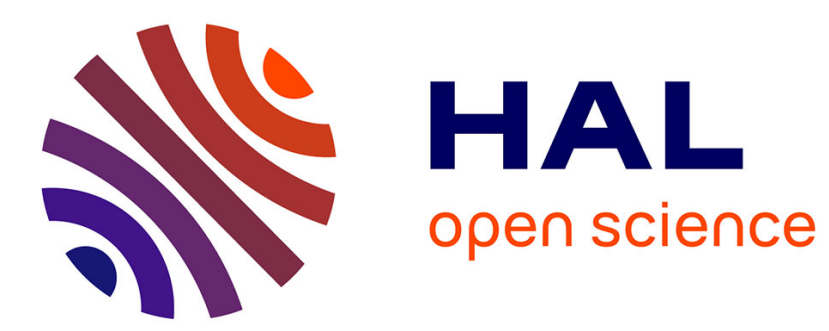

\title{
De la bibliothèque au rond-point : la patrimonialisation fragmentée de Robert Pinget
}

\author{
Clothilde Roullier
}

\section{To cite this version:}

Clothilde Roullier. De la bibliothèque au rond-point : la patrimonialisation fragmentée de Robert Pinget. Culture et Musées, 2021, 38, pp.303-306. 10.4000/culturemusees.7383 . hal-03471627

\section{HAL Id: hal-03471627 \\ https://hal.science/hal-03471627}

Submitted on 8 Dec 2021

HAL is a multi-disciplinary open access archive for the deposit and dissemination of scientific research documents, whether they are published or not. The documents may come from teaching and research institutions in France or abroad, or from public or private research centers.
L'archive ouverte pluridisciplinaire HAL, est destinée au dépôt et à la diffusion de documents scientifiques de niveau recherche, publiés ou non, émanant des établissements d'enseignement et de recherche français ou étrangers, des laboratoires publics ou privés. 


\title{
OpenEdition
}

Journals

\section{Culture \& Musées}

Muséologie et recherches sur la culture

$38 \mid 2021$

Patrimonialisations de la littérature

Études \& notes de recherche

\section{De la bibliothèque au rond-point : la patrimonialisation fragmentée de Robert Pinget}

\author{
Clothilde Roullier
}

p. 303-306

https://doi.org/10.4000/culturemusees.7383

\section{Entrées d'index}

Rubriques: Notes de recherche

\section{Texte intégral}

La postérité de l'écrivain franco-suisse Robert Pinget (1919-1997) se partage entre différents lieux de mémoire des institutions (bibliothèques, musées, services d'archives, etc.) et des espaces géographiques (Paris et province, pays étrangers). Pinget est un auteur qui a fait carrière en France. Il est né à Genève et, après avoir fait des études de droit pour devenir avocat, est parti à Paris après la Seconde Guerre mondiale pour étudier à l'École nationale supérieure des beaux-arts. N'ayant jamais vraiment cessé d'écrire depuis l'adolescence, il est finalement devenu écrivain et, à partir de 1957, a fait partie du Nouveau Roman orchestré par Alain Robbe-Grillet aux Éditions de Minuit.

2 Il n'existe pas de maison-musée dédiée à l'auteur, qui a vécu à partir de 1965 une grande partie du temps en Touraine, près de Loches, mais seulement une rue à Loches ainsi qu'un rond-point et une portion de route à Tours portant son nom ${ }^{1}$. Ce rond-point est entré dans l'histoire, si l'on peut dire, le 24 octobre 2014, jour où l'écrivain François Bon a tenté de l'activer à travers une performance vidéo tout en montrant son inanité2. Resté sur le rond-point, il n'a pas emprunté la rue Robert-Pinget qui en part et qui aboutit rue Albert-Camus, heureux hasard pourtant, puisque Camus a, en 1953, 
soutenu Pinget dans son début de carrière aux Éditions Gallimard lors de la publication du Renard et la Boussole? .

3 Entre cette absence de centre qui aurait pu être consacré à l'écrivain (en particulier dans sa maison en Touraine qu'il avait remaniée en la flanquant d'une tour baptisée du nom d'un de ses personnages de roman : la tour Mahu) et cette inscription relativement arbitraire dans une infrastructure routière, de multiples conservatoires de traces existent en réalité, qu'une double enquête biographique et institutionnelle permet progressivement de mettre au jour4.

\section{Le lieu du corps}

La trajectoire de l'auteur, né à Genève en 1919 et arrivé à Paris en 1946 pour y étudier à l'École des beaux-arts, explique en partie le phénomène de fragmentation des traces. Partagé entre deux pays, pratiquement entre deux vies aussi (celle des relations familiales durables malgré l'éloignement géographique et l'affirmation d'une voie hors du cadre établi dans son milieu d'origine, et celle de la singularité et des relations choisies), Pinget a, après sa mort à Tours en 1997, d'abord été partagé en deux par le notaire : une partie des cendres est allée à sa famille, l'autre à son héritier. Celles-ci reposent aujourd'hui pour moitié dans le caveau familial à Compesières, dans le canton de Genève, et, pour l'autre moitié, ont été dispersées en surface du même caveau après avoir transité par le Sénégal pendant quelques années.

$5 \quad$ Si le corps atomisé, pratiquement réuni, est en Suisse, l'œuvre, avec ses traces, est davantage à chercher en France. Pour autant, l'entièreté de la production de l'auteur est difficilement appréhendable. Ce dernier étant essentiellement connu comme écrivain et non comme peintre, ses manuscrits sont, depuis 2003, conservés, sanctuarisés, à la bibliothèque littéraire Jacques-Doucet, à Paris, avec une très petite partie de ses tableaux et dessins 5 . S'agissant de l'œuvre peint, il s'en est fallu de peu pour qu'un grand musée français fasse l'acquisition de tableaux retrouvés chez un brocanteur en 2014, mais ce ratage a ré-ancré pour quelque temps encore l'image de l'auteur dans une dimension strictement scripturale. Quelques autres dessins et peintures ont fait l'objet d'enchères en salles des ventes ou sur Internet, mais la part de ce qui est inventoriable à partir de ces sources demeure très faible par rapport à ce qui a été produit, car Pinget, bien qu'ayant renoncé dès les années 1950 à une carrière de peintre, n'a en réalité jamais cessé de peindre et de dessiner. À ce jour, seul un relevé des dessins figurant dans les manuscrits a, à notre connaissance, été réalisé par le bibliothécaire qui a inventorié le fonds à la bibliothèque littéraire Jacques-Doucet.

\section{La dimension de l'œuvre}

$6 \quad$ En ce qui concerne les traces strictement littéraires (bibliothèque personnelle et manuscrits, lecture et écriture), elles se répartissent entre trois espaces géographiques associés à la vie de l'auteur : Genève, Paris et Tours. C'est dans la capitale que se confondent à la fois les années décisives de formation avant l'achat de la maison en Touraine, un attachement à l'appartement du 4, rue de l'Université, acquis pour lui par ses parents en 1953 (il était auparavant, depuis 1949, locataire au 82, rue de Rennes), et l'institutionnalisation (discrète) de l'auteur, aussi bien pré que post-mortem, passant des Éditions de Minuit, 7, rue Bernard-Palissy, à la bibliothèque littéraire JacquesDoucet, 8, place du Panthéon. Dans cette grande bâtisse virtuelle que forment les 5e, $6 \mathrm{e}$ et 7 e arrondissements de Paris, on a presque la même configuration que celle défendue par Jean-Paul Dekiss (2009) pour la maison de Jules Verne à Amiens, avec le salon de l'éditeur Pierre-Jules Hetzel reproduit au premier étage de la maison de l'écrivain : à condition de mêler les temporalités, le bureau de l'éditeur, l'appartement de l'auteur et le « reposoir » de l'œuvre se trouvent ici réunis de façon cohérente.

Cette cohérence ne doit toutefois pas masquer un éparpillement relatif des sources. En effet, Pinget a, de son vivant, en 1993, prêté une partie de ses manuscrits pour 
microfilmage à la bibliothèque municipale de Tours. Bien que ces manuscrits fassent pratiquement tous partie aujourd'hui du fonds Pinget conservé à la bibliothèque littéraire Jacques-Doucet, on relève quelques lacunes en divers endroits si l'on compare minutieusement les inventaires. Quant à Genève, on y trouvera uniquement des photocopies d'une partie des mêmes manuscrits, données en 2006 à la bibliothèque de la ville. La difficulté pour les manuscrits se concentre donc autour de l'identification des manques et redondances entre Tours et Paris.

8 Si l'on prend également en compte le premier volet du diptyque lecture/écriture, à savoir les livres possédés et éventuellement annotés par l'auteur, les descriptifs des fonds conservés par les institutions sont en partie muets sur le sujet, alors qu'une partie de la bibliothèque de Pinget est également conservée à la bibliothèque littéraire Jacques-Doucet et que la bibliothèque municipale de Tours détient, sous forme de photocopies, une part importante de ses annotations manuscrites. Au vu du relevé établi par deux chercheurs dans ses deux domiciles après son décès (à Paris et à Luzillé, en Touraine) (Liéber \& Renouard, 2001), nous constatons que l'entièreté de la bibliothèque de l'auteur n'est pas reconstituable à partir des exemplaires et photocopies disponibles : trop d'ouvrages sont manquants dans les inventaires (non publiés). Seul le recours aux humanités numériques ${ }^{6}$ permettrait alors de rendre accessible l'ensemble des ressources disponibles à ce propos, en croisant le relevé des livres possédés avec, par exemple, une numérisation des ouvrages dans l'édition de référence pour ceux qui n'étaient pas annotés, et une numérisation des annotations pour les autres.

\section{Vers une compréhension globale du processus de patrimonialisation}

Le fragment et la ligne de partage traversent toute l'histoire de la patrimonialisation de Pinget, du fait de sa propre volonté (le choix des manuscrits proposés, de son vivant, pour microfilmage relève de cette catégorie) ou non (depuis le passage de la frontière en 1946, avant d'avoir produit une œuvre, jusqu'aux transferts et partages opérés après sa mort). Nous pourrions ainsi tenter de retracer toutes les lignes de partage ayant potentiellement eu une incidence sur les restes disponibles post-mortem, aboutissant à une complexe carte d'identité de sa production littéraire et artistique. Pinget est un auteur qui a poussé loin l'expérience littéraire, frôlant sans doute, dans son exercice comme dans ses résultats, ce que Barthes affirmait comme une évidence dans son article « La mort de l'auteur » : l'écriture dissout « toute identité », y compris celle « du corps qui écrit » (Barthes, 1968 : 12). L'écrivain naît dans le livre à la place de l'auteur, et " il n'y a de biographie que de la vie improductive », puisque " dès que je produis, dès que j'écris, c'est le Texte lui-même qui me dépossède (heureusement) de ma durée narrative " (Barthes, $1975: 6$ ). Nous rejoignons là une des ritournelles de Pinget,

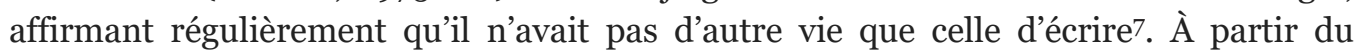
devenir artiste-écrivain de Pinget (disons 1946), il y aurait donc, si l'on fait converger la signification même de l'œuvre avec ses inscriptions matérielles, interagissant avec les acteurs et les institutions, une mise en pièces à déterrer et déchiffrer pour faire apparaître la patrimonialisation en acte : son processus de transmission inscrit dans le vivant.

\section{Bibliographie}

Barthes (Roland). 1968. « La mort de l'auteur ». Mantéia, 5, p. 12-17.

Barthes (Roland). 1975. Roland Barthes par Roland Barthes. Paris : Seuil (Écrivains de toujours).

Dekiss (Jean-Paul). 2009. " La maison d'un écrivain, utopie ou enjeu de société ». Revue d'histoire littéraire de la France, 109(4), p. 783-795, en ligne : https://www.cairn.info/revue-dhistoire-litteraire-de-la-france-2009-4-page-783.htm [consulté le 28 juin 2021].

DOI : 10.3917/rhlf.094.0783 
Liéber (Jean-Claude) \& Renouard (Madeleine). 2001. « La bibliothèque de monsieur Songe : les livres de Robert Pinget », p. 225-255 in Bibliothèques d'écrivains / sous la direction de Paolo D’Iorio et Daniel Ferrer. Paris : CNRS Éditions (Textes et manuscrits).

\section{Sitographie}

« Les bibliothèques d'artistes », en ligne : http://www.lesbibliothequesdartistes.org/ (consulté le 28 juin 2021).

«Calames (catalogue des archives et des manuscrits de l'enseignement supérieur) », en ligne : http://www.calames.abes.fr/pub (consulté le 28 juin 2021).

Bibliothèque littéraire Jacques-Doucet, fonds Robert Pinget, en ligne : http://www.calames.abes.fr/pub/bljd.aspx\#details?id=FileId-311 (consulté le 28 juin 2021).

«Le Tiers Livre », site de François Bon, en ligne : https://www.tierslivre.net/spip/spip.php? article4049 (consulté le 28 juin 2021).

\section{Notes}

1 Notons que le rond-point Robert-Pinget, à Tours, quartier Saint-Symphorien, a changé de place entre 1999 et 2010 : originellement situé entre le boulevard Abel-Gance et une voie de raccordement en direction de l'autoroute A10 (délibération du 11 octobre 1999), le conseil municipal a finalement décidé de «l'extension de l'appellation rue Daniel-Mayer depuis le boulevard Abel-Gance jusqu'au à la rue des Bordiers » et de " la dénomination rond-point Daniel-Mayer en substitution du rond-point actuel Robert-Pinget », faisant que l'appellation rond-point Robert-Pinget s'applique désormais à celui joignant la rue Daniel-Mayer et la rue Robert-Pinget, ladite rue se terminant là où commence la rue Albert-Camus (délibération du 31 mai 2010).

2 Voir, en ligne : https://www.tierslivre.net/spip/spip.php?article4049 (consulté le 28 juin 2021).

3 La correspondance croisée entre les deux écrivains, pour les années 1953-1956, est conservée à la bibliothèque littéraire Jacques-Doucet (fonds Pinget, sous forme de photocopies) et au Centre de documentation Albert-Camus, à Aix-en-Provence.

4 Cette recherche s'inscrit dans les marges d'une biographie de Robert Pinget en cours d'écriture.

5 Voir, en ligne : http://www.calames.abes.fr/pub/bljd.aspx\#details?id=FileId-311 (consulté le 28 juin 2021).

6 Dans le même esprit que sur le site en ligne : http://www.lesbibliothequesdartistes.org/ (consulté le 28 juin 2021).

7 Voir notamment son entretien avec Louis-Albert Zbinden, Gazette de Lausanne, 4 décembre 1965, p. 18.

\section{Pour citer cet article}

Référence papier

Clothilde Roullier, « De la bibliothèque au rond-point : la patrimonialisation fragmentée de Robert Pinget », Culture \& Musées, 38 | 2021, 303-306.

Référence électronique

Clothilde Roullier, « De la bibliothèque au rond-point : la patrimonialisation fragmentée de Robert Pinget », Culture \& Musées [En ligne], 38 | 2021, mis en ligne le 10 novembre 2021, consulté le 08 décembre 2021. URL : http://journals.openedition.org/culturemusees/7383; DOI : https://doi.org/10.4000/culturemusees.7383

\section{Auteur}

\section{Clothilde Roullier}

Archives nationales

Clothilde Roullier est docteure ès lettres, chargée d'études documentaires aux Archives nationales, responsable des fonds relatifs à la création artistique. Elle est également chercheure associée au laboratoire Art des images et art contemporain de l'Université Paris-8. Elle a édité La Fissure précédé de Malicotte-la-Frontière de Robert Pinget (Genève, MétisPresses, 2009) et, en collaboration avec Patrick Suter, un dossier d'inédits du même auteur dans la Revue des sciences humaines $\left(n^{\circ} 317,2015\right)$. Elle a notamment publié « Robert Pinget, guide des sources ou tentative d'épuisement des traces d'une histoire littéraire du Xxe siècle » (Histoires littéraires, $n^{\circ} 40$, octobre-décembre 2009) et «Pinget traqué : trouvailles et bizarreries du repérage archivistique » (dans Robert Pinget. Matériau, marges, écriture, sous la direction de Martin Mégevand et Nathalie Piégay-Gros, Presses universitaires de Vincennes, coll. « Manuscrits 
modernes ", 2011).

Courriel : clothilde.roullier[at]culture.gouv.fr

\section{Droits d'auteur}

Culture \& Musées 